\title{
Desempenho de Pessoas com Autismo em Tarefas de Emparelhamento com o Modelo por Identidade: Efeitos da Organização dos Estímulos
}

\author{
Effects of Stimuli Organization on Identity Matching-to-Sample Performances \\ of Persons with Autism
}

\author{
Camila Graciella Santos Gomes ${ }^{* 1}$ \& Deisy das Graças de Souza** \\ Universidade Federal de São Carlos, São Carlos, Brasil
}

\begin{abstract}
Resumo
O estudo avaliou o desempenho de 20 pessoas com autismo em uma tarefa de emparelhamento por identidade sob dois procedimentos que diferiram quanto à forma de apresentação dos estímulos e à resposta de escolha. No procedimento de matching típico cada tentativa apresentava um estímulo modelo e três de comparação; no matching adaptado três modelos e três estímulos de comparação eram apresentados simultaneamente. $\mathrm{O}$ procedimento foi conduzido em três blocos: 10 tentativas de matching adaptado, 10 de matching típico e 20 tentativas com os dois arranjos misturados. A média de acertos foi significativamente maior no matching adaptado, mas ocorreu acentuada variabilidade inter-individual e esse arranjo favoreceu principalmente o desempenho de participantes com escores menores. A história prévia de aprendizagem dos participantes, entre outros fatores, pode ter influenciado nesses resultados, o que requer melhor avaliação, com outros controles experimentais. Palavras-chave: Relações condicionais; emparelhamento com o modelo; emparelhamento de identidade; formação de conceitos; autismo.
\end{abstract}

\begin{abstract}
This study assessed the performance of 20 persons with autism in an identity matching-to-sample task, using two procedures with different stimuli arrangements and different response requirements. The typical matching procedure presented one sample stimulus and three comparison stimuli; the adapted matching displayed three sample stimuli and three comparison stimuli simultaneously. Three consecutive sets of trials were conducted: a 10-trial set of adapted matching, a 10-trial set of typical matching, and a 20-trial set intermixing both trial types in an unsystematic order. The average score of correct performance was significantly higher under the adapted matching than under the typical matching, but the inter-individual variability was large and the adapted arrangement favored mainly the performance of participants with lower scores. The participants' previous history in learning may have played a role in the way they solved the present task, but further investigation, with experimental controls additional will be necessary to evaluate this possibility.

Keywords: Conditional relations; identity matching-to-sample; concept formation; autism.
\end{abstract}

\footnotetext{
* Endereço para correspondência: Avenida Álvares Cabral, 1030, sala 506, Lourdes, Belo Horizonte, MG, 30170-001. E-mail: camilagsg@uol.com.br

*** Endereço para correspondência: Universidade Federal de São Carlos, Centro de Educação e Ciências Humanas, Via Washington Luis, KM 235, Monjolinho, São Carlos, SP, 13565905. Caixa-Postal: 676.E-mail: ddgs@ power.ufscar.br ${ }^{1}$ Este artigo é baseado no primeiro de dois estudos conduzidos pela primeira autora como parte das exigências para obtenção do título de Mestre no Programa de Pós-Graduação em Educação Especial da Universidade Federal de São Carlos, com apoio da Coordenação de Aperfeiçoamento de Pessoal de Nível Superior (Capes). A preparação do manuscrito contou com apoio do Programa de Apoio a Núcleos de Excelência (PRONEX), Fundação de Amparo à Pesquisa do Estado de São Paulo (FAPESP) / Conselho Nacional de Desenvolvimento Científico e Tecnológico (CNPq) (Processo No. 2003/ 09928-4).

Agradecemos às Dras. Maria Amelia Almeida, Ana Lúcia R Aiello, Carolina Lampreia e a dois revisores anônimos pela leitura cuidadosa e pelas sugestões para aprimoramento do texto; à Rafiza Drumond C. Lobato e às escolas ComumViver e DiaDia pela colaboração durante a coleta dos dados. E agradecemos, especialmente, aos participantes e suas famílias pela generosidade em participar da investigação.
}

Tarefas de emparelhamento com o modelo (matchingto-sample ou MTS) são muito utilizadas para o ensino e a avaliação de relações condicionais, ou seja, relações entre estímulos, que podem dar origem à formação de classes de equivalência (Sidman \& Tailby, 1982) e ao comportamento simbólico.

No emparelhamento com o modelo típico, a tarefa é constituída por uma sequiência ou conjunto de tentativas discretas, planejadas para ensinar relações entre dois conjuntos de estímulos, o conjunto dos estímulos modelo e o conjunto dos estímulos de comparação ou escolha. Em cada tentativa são apresentados um estímulo modelo e dois ou mais estímulos de comparação. Para cada modelo, há apenas um estímulo de comparação correto, referido com estímulo discriminativo ou $\mathrm{S}+\mathrm{e}$ todos os outros estímulos de comparação são incorretos, referidos como estímulos negativos ou S-. Ao longo da série de tentativas, os diferentes estímulos modelo vão se alternado e o estímulo de compa- 
ração correto é aquele que se pretende que o aprendiz relacione ao estímulo modelo presente. Esta característica do procedimento requer especial atenção do aprendiz para ambos os estímulos, uma vez que a seleção de um estímulo de comparação sem considerar o estímulo modelo pode levar a erro. Assim, a escolha de um estímulo que era o correto em uma tentativa, não garante que a escolha do mesmo estímulo será correta na tentativa seguinte ou em outras tentativas. Em situação de ensino, as respostas de escolha são seguidas por conseqüências diferenciais, ou seja, repostas corretas são reforçadas e escolhas incorretas não são seguidas de reforço (Saunders \& Green, 1999).

O grau de correspondência entre modelos e comparações pode variar ao longo de um contínuo que inclui desde relações de identidade até relações puramente arbitrárias (de Rose, 2004). Quando a correspondência é baseada em identidade, um dos estímulos de comparação é igual ao estímulo modelo e os outros são diferentes. A tarefa do aprendiz é escolher, para cada modelo, o comparação idêntico e a tarefa é denominada matching de identidade. O ensino sistemático de relações de identidade pode dar origem ao desempenho de identidade generalizado, isto é, o indivíduo aprende a relacionar elementos iguais, mesmo que nunca tenha tido qualquer tipo de experiência prévia com eles, quando então se pode dizer que ele formou o conceito de igualdade (Catania, 1999; Dube, 1996; Serna, Dube, \& McIlvane, 1997). Quando o emparelhamento com o modelo é arbitrário, não há similaridade entre modelo e comparação e a correspondência entre eles é baseada em relações inteiramente arbitrárias ou convencionais como, por exemplo, no caso de relações entre objetos e seus nomes, que variam de acordo com as convenções de cada comunidade verbal (de Rose, 2004; Dube, 1996).

Tarefas de emparelhamento com o modelo podem empregar estímulos de diferentes naturezas e que afetam diferentes modalidades sensoriais, dependendo de quais relações se pretende ensinar. Por exemplo, os estímulos podem ser auditivos, como sons e palavras ditadas, ou visuais, como figuras e palavras impressas, e as relações estabelecidas entre eles podem ser visuais-visuais, isto é, na mesma modalidade sensorial, ou auditivo-visuais, o que implica diferentes modalidades sensoriais (Dube, Green, \& Serna, 1993); mas nada impede que sejam ensinadas relações nas quais um dos conjuntos inclua estímulos olfativos (Annet \& Leslie, 1995), táteis (Belanich \& Fields, 1999), gustativos (Hayes, Tilley, \& Hayes, 1988) e até mesmo estímulos proprioceptivos (DeGrandpre, Bickel, \& Higgins, 1992). Considerando a forma de apresentação dos estímulos, o emparelhamento com o modelo pode ser simultâneo ou com atraso. No matching simultâneo, como o próprio nome sugere, os estímulos modelo e os de comparação são apresentados simultaneamente, enquanto no matching com atraso, uma resposta ao estímulo modelo produz a remoção desse estímulo e a apresentação dos estímulos de comparação que, então, ficam disponíveis para escolha, na ausência do modelo (Gutowski \& Stromer, 2003).

Dada a eficácia do emparelhamento com o modelo para ensinar relações entre estímulos e, sobretudo para engen- drar a emergência de relações novas, não diretamente ensinadas, derivadas de um conjunto de relações ensinadas (Sidman, 1971, 1985, 1994; Sidman \& Tailby, 1982), seu emprego torna-se especialmente importante para o ensino de indivíduos com necessidades especiais, entre os quais indivíduos com autismo. Apesar do uso amplamente disseminado do procedimento de emparelhamento com o modelo típico em situações de aplicação, com diversas populações (cf. Ghezzi, Williams, \& Carr, 1999; Martin \& Pear, 1978), a literatura especializada tem descrito dificuldades no ensino de relações condicionais a pessoas com autismo (eg., Eikeseth \& Smith, 1992; Kelly, Green, \& Sidman, 1998; Williams, Perez-González, \& Queiroz, 2005). No estudo de Eikeseth e Smith (1992), por exemplo, o emparelhamento com o modelo foi empregado para ensinar, a autistas de alto funcionamento, relações arbitrárias envolvendo estímulos visuais. A quantidade de tentativas necessárias para que os participantes aprendessem as relações ensinadas na primeira fase do estudo variou entre $306 \mathrm{e}$ 1.546. Os pesquisadores sugeriram que o elevado número de tentativas de treino poderia ser indício de problemas no procedimento de ensino e apontaram para a necessidade de se buscar outras formas mais eficientes de ensinar, como por exemplo, por meio do procedimento de ensino por blocos desenvolvido Saunders e Spradlin (1989).

Esse procedimento, desenvolvido com dois adultos com retardo mental severo que vinham apresentando dificuldades em aprender relações arbitrárias em tarefas de emparelhamento com o modelo, resultou de uma análise dos componentes do emparelhamento arbitrário e do planejamento de uma seqüência de ensino para cada um dos componentes. Era conduzido um bloco de tentativas consecutivas com um mesmo estímulo modelo, seguido por um bloco de tentativas consecutivas com outro estímulo modelo; essa alternância continuava até que o participante diminuísse o número de erros; então, o número de tentativas por bloco com um mesmo estímulo modelo diminuía, ao mesmo tempo em que aumentava-se a freqüência de alternância entre os blocos até que, finalmente, a apresentação dos estímulos modelos passava a ser randomizada, sem que os participantes cometessem erros na seleção do estímulo de comparação correto. O estudo mostrou que os participantes não só aprenderam as discriminações iniciais, mas também aprenderam novas relações com maior rapidez (Saunders \& Spradlin, 1990).

Uma adaptação do procedimento de Saunders e Spradlin $(1989,1990)$ foi empregada por Williams et al. (2005) para ensinar relações auditivo-visuais entre cores (branco e preto) e os nomes correspondentes a um adolescente com autismo. Neste estudo foram necessárias 792 tentativas de treino para que o participante aprendesse a discriminar entre as duas cores.

Em outro estudo que visava ensinar e avaliar relações de identidade entre estímulos visuais e relações arbitrárias auditivo-visuais com uma criança com autismo, Kelly et al. (1998) verificaram que o desempenho do participante nas tarefas de matching de identidade variava de acordo com a maneira pela qual as tarefas eram apresentadas (usan- 
do um computador ou material concreto) e também, de acordo com o número de estímulos de comparação presentes. Os autores concluíram que o baixo desempenho da criança em algumas tarefas provavelmente resultou dos procedimentos utilizados, tanto no ensino quanto nos testes. Com base nesses resultados os autores sugeriram que, em investigações sobre controle de estímulos, os pesquisadores questionem seus procedimentos quando o participante não exibir um tipo específico de controle de estímulos que se pretendia estabelecer. Em outras palavras, antes de concluir, apressadamente, que o indivíduo não aprende, é preciso perguntar se não é o procedimento que não ensina (cf. Keller, 1968; Sidman, 1985).

O programa TEACCH (Treatment and Education of Autistic and Communication Handicapped Children), desenvolvido por Schopler na década de 1970 na Universidade da Carolina do Norte e utilizado para educar pessoas com autismo em muitos países, inclusive no Brasil, emprega, como recurso para o ensino de relações condicionais, tarefas de emparelhamento com o modelo adaptadas, de acordo com a metodologia geral utilizada pelo programa (Lewins \& de Leon, 1995; Mesibov, Schopler, \& Hearsey, 1994; Tulimoschi, 2002).

A adaptação leva em consideração alguns aspectos da aprendizagem de pessoas com autismo e critérios de educação estruturada e, diferentemente do matching típico ou padrão, que apresenta um estímulo modelo e dois ou mais estímulos de comparação, o matching adaptado apresenta o mesmo número de estímulos modelos e comparações simultaneamente (TEACCH, 1992). A principal justificativa para esta adaptação é que pessoas com autismo parecem responder melhor a tarefas que sejam visualmente lógicas, ou seja, tarefas em que a própria organização dos estímulos indique ao aprendiz o que ele deve fazer sem a necessidade de muitas instruções verbais. Além disso, o fato de emparelhar os estímulos um a um, até completar todos os pares, torna a atividade mais óbvia, indicando visualmente o término da tarefa, uma vez que nenhum estímulo fica "sobrando", sem um par (Mesibov et al., 1994; Peetres, 1998).

Apesar do matching adaptado ser aparentemente adequado às características de aprendizagem de pessoas com autismo e de ser bastante utilizado em instituições que empregam a metodologia do programa TEACCH, a literatura não registra dados de estudos empíricos para a verificação de sua efetividade, nem de sua contribuição relativa, em comparação com o matching típico. Por esse motivo, o objetivo deste estudo foi avaliar o desempenho de participantes com autismo em tarefas de matching de identidade no procedimento típico e no procedimento adaptado.

\section{Método}

\section{Participantes}

Realizaram as tarefas propostas vinte pessoas com diagnóstico de autismo, cinco mulheres e quinze homens, com idades entre quatro anos e onze meses e trinta e um anos. Sete participantes estavam matriculados em escolas regu- lares e treze em escolas especiais. Onze utilizavam a fala para se comunicar e nove não falavam. A Tabela 1 apresenta a lista de participantes e suas principais características.

Os critérios de seleção dos participantes incluíram diagnóstico prévio de autismo e disponibilidade da família e da escola para a participação do aluno na pesquisa; além disso, procurou-se assegurar que os participantes não tivessem experiência prévia de treino rotineiro no mesmo tipo de tarefa. As escolas especiais escolhidas para a avaliação não utilizavam o programa TEACCH de maneira sistemática, apesar de já terem utilizado ou ainda utilizarem algumas estratégias de ensino com base no programa. Tanto o recrutamento dos participantes quanto os demais procedimentos empregados no estudo foram aprovados pelo Comitê de Ética em Pesquisa em Seres Humanos da Universidade Federal de São Carlos, para um projeto mais amplo de ensino de relações condicionais a pessoas com autismo (Parecer número 234/2006).

Antes do início do procedimento com as tarefas de emparelhamento os participantes foram avaliados com referência na escala CARS - Childhood Autism Rating Scale (Schopler, Reichler, \& Renner, 1988). Este instrumento permite identificar pessoas com características comportamentais de autismo e distinguir entre autismo e atraso no desenvolvimento sem autismo. De acordo com a escala, os resultados da avaliação podem ser distribuídos em três categorias: desenvolvimento normal, autismo leve/moderado e autismo grave. Apesar de ser direcionada para a avaliação do desenvolvimento infantil, esta escala foi utilizada neste estudo com pessoas de até dezesseis anos para se obter referência a respeito de seus repertórios, devido à facilidade de sua utilização e à falta de outra escala mais adequada. Os participantes com idade acima de 16 anos não foram avaliados pela escala, mas tinham diagnóstico médico de autismo relatado pela família ou registrado no prontuário escolar. Os participantes com Síndrome de Asperger também não foram avaliados pela CARS, pois apesar deste transtorno ser considerado dentro de um contínuo autístico (Wing, 1988), a escala não é adequada para avaliar esse quadro.

\section{Situações e Materiais}

Sessões individuais foram conduzidas em uma sala de um consultório particular para atendimento educacional ou em uma sala de aula da escola que os participantes freqüentavam, devidamente reservada para a avaliação e adequada às necessidades da pesquisa. As salas eram mobiliadas com uma mesa, duas cadeiras e uma filmadora.

O participante sentava-se à mesa, voltado para a parede e a pesquisadora permanecia atrás dele. Em algumas situações na escola especial estava presente um professor ou acompanhante do participante. Em geral, cada participante realizou as tarefas em uma única sessão, que teve entre quinze e trinta minutos de duração.

Todas as tentativas das tarefas de emparelhamento com o modelo foram montadas em divisórias de fichário na cor branca, de $21 \mathrm{~cm}$ x $30 \mathrm{~cm}$ e organizadas seqüencialmente em um fichário. As divisórias serão denominadas páginas, 
Gomes, C. G. S. \& de Souza, D. G. (2008). Desempenho de Pessoas com Autismo em Tarefas de Emparelhamento com o Modelo por Identidade: Efeitos da Organização dos Estímulos.

Tabela 1

Características Gerais dos Participantes: Idade, Gênero, Categorização Segundo a CARS, Presença ou Não de Repertório Verbal, Tipo de Escola Freqüentada e Diagnóstico Associado

\begin{tabular}{|c|c|c|c|c|c|c|}
\hline Participante & Idade & Sexo & CARS & Verbal & Tipo de Escola & Diagnóstico Associado ${ }^{a}$ \\
\hline $\mathrm{P} 1$ & $12 \mathrm{a}$ & feminino & leve/moderado & $\operatorname{sim}$ & regular & \\
\hline $\mathrm{P} 2$ & 6a $3 \mathrm{~m}$ & masculino & grave & $\operatorname{sim}$ & regular & Psicose \\
\hline $\mathrm{P} 3$ & 9a $10 \mathrm{~m}$ & masculino & leve/moderado & $\operatorname{sim}$ & regular & \\
\hline P4 & $6 \mathrm{a} 5 \mathrm{~m}$ & masculino & ------------------- & $\operatorname{sim}$ & regular & Síndrome de Asperger \\
\hline P5 & $9 \mathrm{a} 5 \mathrm{~m}$ & feminino & grave & não & especial & Psicose \\
\hline P6 & $13 \mathrm{a}$ & masculino & grave & não & especial & Surdez \\
\hline P7 & $9 \mathrm{a} 10 \mathrm{~m}$ & feminino & leve/moderado & $\operatorname{sim}$ & especial & $\begin{array}{l}\text { Transtorno de déficit } \\
\text { de atenção e hiperatividade }\end{array}$ \\
\hline P8 & $16 \mathrm{a} 5 \mathrm{~m}$ & masculino & grave & não & especial & \\
\hline P9 & $10 \mathrm{a} 8 \mathrm{~m}$ & masculino & grave & $\operatorname{sim}$ & regular & \\
\hline P10 & $31 \mathrm{a}$ & feminino & ------------------- & $\operatorname{sim}$ & especial & \\
\hline P11 & $11 \mathrm{a} 6 \mathrm{~m}$ & masculino & leve/moderado & $\operatorname{sim}$ & especial & \\
\hline $\mathrm{P} 12$ & $11 \mathrm{a} 2 \mathrm{~m}$ & masculino & leve/moderado & não & especial & \\
\hline P13 & 11a $2 \mathrm{~m}$ & masculino & leve/moderado & não & especial & \\
\hline P14 & 11a $10 \mathrm{~m}$ & masculino & leve/moderado & $\operatorname{sim}$ & especial & \\
\hline P15 & $22 \mathrm{a} 9 \mathrm{~m}$ & masculino & 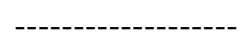 & $\operatorname{sim}$ & especial & Síndrome de Asperger \\
\hline P16 & 6a $5 \mathrm{~m}$ & masculino & leve/moderado & não & regular & \\
\hline P17 & 4a $11 \mathrm{~m}$ & masculino & leve/moderado & $\operatorname{sim}$ & regular & \\
\hline $\mathrm{P} 18$ & $25 \mathrm{a} 9 \mathrm{~m}$ & masculino & 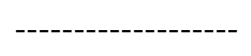 & não & especial & \\
\hline P19 & $13 \mathrm{a} 3 \mathrm{~m}$ & feminino & leve/moderado & $\operatorname{sim}$ & regular & Autismo de alto funcionamento \\
\hline $\mathrm{P} 20$ & $18 \mathrm{a} 4 \mathrm{~m}$ & masculino & 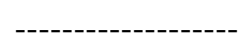 & não & especial & \\
\hline
\end{tabular}

Nota. ${ }^{a} \mathrm{O}$ diagnóstico foi localizado nos prontuários dos participantes para os quais havia algum tipo de avaliação especializada disponível.

para conveniência de expressão. O fichário era apresentado aberto, de modo que duas páginas ficavam à mostra, uma à esquerda e outra à direita. Utilizou-se também figuras, velcro e palitos de madeira. As figuras usadas nos dois tipos de tarefas de emparelhamento eram fotografias, desenhos coloridos, pictogramas em preto e branco, palavras impressas, sequiências de letras e figuras compostas por números e quantidades.

No matching típico cada tentativa apresentava um estímulo modelo fixo na parte superior da página à direita $\mathrm{e}$ três estímulos de comparação fixos na parte inferior da mesma, como mostra a Figura 1 (Painel A). Na página à esquerda ficava um palito de madeira, preso com velcro (sinalizador). Abaixo de cada estímulo de comparação havia também um pedaço de velcro no qual o participante podia prender o sinalizador para indicar sua escolha.

No matching adaptado cada tentativa apresentava três estímulos modelos fixos na página da direita e três estímulos de comparação móveis, que tinham velcro na parte de atrás e eram apresentados presos ao velcro da página à esquerda; esses estímulos podiam ser removidos e pregados sobre cada um dos estímulos modelos da página à direita, que tinham pequenos pedaços de velcro na parte superior e inferior, como mostra a Figura 1 (Painel B).
Todas as tentativas das tarefas de emparelhamento com o modelo eram organizadas da esquerda para a direita: com esse arranjo visual o participante iniciava a atividade pegando os estímulos que ficavam presos à esquerda com velcro (o sinalizador, no caso do matching típico, e figuras, no caso do matching adaptado) e, em seguida, colocava-os presos ao velcro dos estímulos à direita (abaixo de um dos estímulos de comparação no matching típico e sobre cada estímulo modelo no matching adaptado). Quando a página da esquerda ficava vazia, isso indicava que aquela tentativa tinha terminado; o participante podia então virar a página e fazer a atividade seguinte (o que se repetia até o término das tentativas).

\section{Procedimento}

A sequiência das tentativas, assim como os estímulos utilizados, eram similares nos dois tipos de emparelhamento. As tarefas, aplicadas em uma única sessão, foram divididas em três blocos de tentativas; o primeiro apresentava tentativas de matching adaptado, o segundo apresentava tentativas de matching típico e o terceiro misturava os dois tipos de tentativas em seqüência aleatória, como resumido na Tabela 2. 

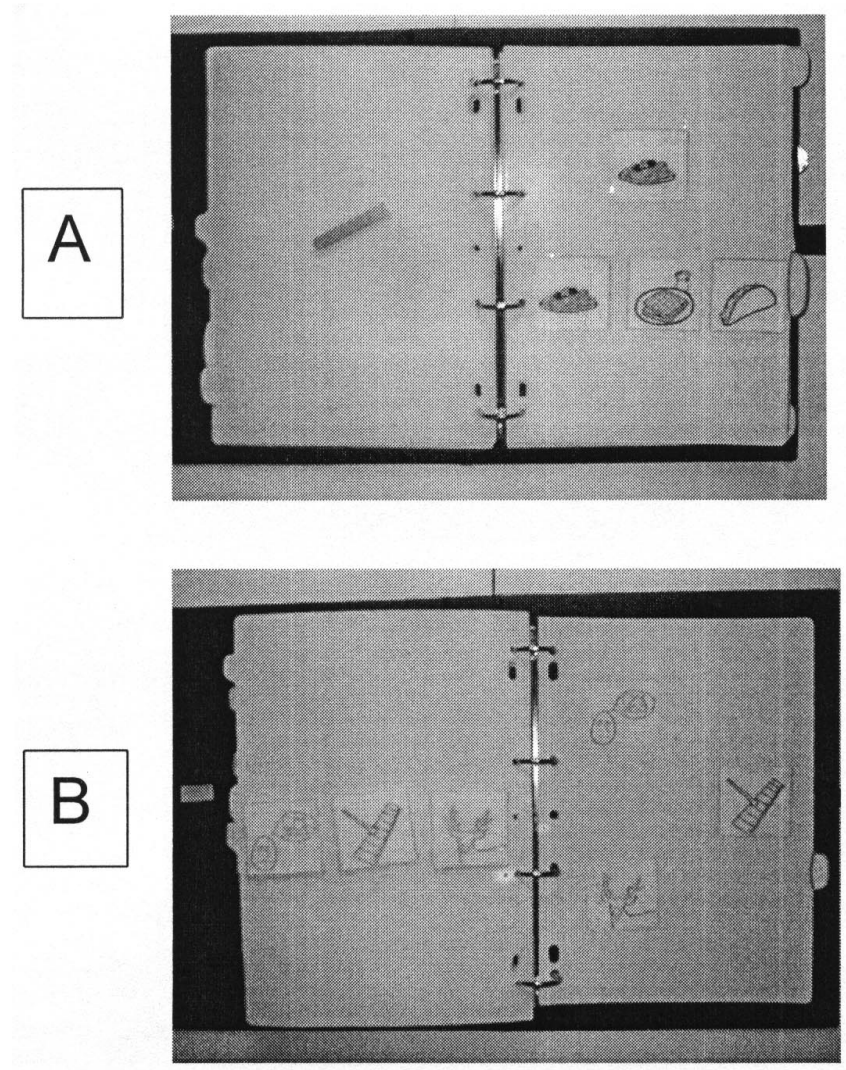

Figura 1. Exemplo de tarefa de emparelhamento por identidade com os arranjos típico (A) e adaptado (B). A. Divisória esquerda: sinalizador (palito de madeira com velcro); divisória direita: o estímulo modelo fixo na parte superior da divisória e três estímulos de comparação fixos na parte inferior, com um pequeno pedaço de velcro exposto; resposta de seleção: pegar o sinalizador à esquerda e colocá-lo sobre o velcro de um dos estímulos de comparação. B. Divisória esquerda: estímulos de comparação presos com velcro; divisória direita: estímulos modelos fixados; resposta de seleção: pegar cada estímulo à esquerda e colocá-lo sobre um dos estímulos à direita

\section{Tabela 2}

Sequiência dos Blocos de Tarefas de Emparelhamento com o Modelo por Identidade

\begin{tabular}{lll} 
Blocos & Tarefas & Número de Tentativas \\
\hline 1. Matching adaptado (A) & Treino & 2 a 10 \\
& Teste & 10 \\
2. Matching típico (B) & Treino & 2 a 10 \\
& Teste & 10 \\
3. Matching típico e adaptado & Teste & 20 \\
em seqüência aleatória & & \\
\hline
\end{tabular}

O primeiro e o segundo blocos incluíam dois tipos de tentativas: tentativas de treino e tentativas de teste (ver Tabela 2). As tentativas de treino, realizadas antes das tentativas de teste, tinham a função de ensinar a tarefa que seria requerida no teste. Nessas tentativas a pesquisadora apresentava instruções verbais e ajuda física, que eram retiradas assim que o participante conseguisse executar a tarefa corretamente. Respostas corretas eram consequenciadas com eventos potencialmente reforçadores, identificados para cada participante, enquanto respostas incorretas eram seguidas de "não" e ajuda física para a correção. O critério para o término do treino e início dos testes era de duas tentativas consecutivas de treino corretas, sem ajuda da pesquisadora, em um único bloco de 10 tentativas. Caso as 10 tentativas fossem completadas sem que o participante apresentasse duas seleções corretas, ele não prosseguia para o teste. Caso as duas tentativas corretas consecutivas ocorressem antes que se completassem 10 tentativas, o treino era interrompido e tinha início imediatamente o teste.

Bloco 1 - Matching Adaptado. Nas 10 tentativas de teste com tarefas de matching adaptado o participante deveria pegar cada um dos três estímulos de comparação à esquerda e colocar sobre um estímulo modelo igual, na página à direita (ver Figura 1B e Tabela 2). O critério para que a tarefa fosse considerada correta era o acerto da relação de identidade entre os três estímulos modelos e seus respectivos estímulos de comparação; caso o participante acertasse apenas uma ou duas das relações, a tentativa era considerada incorreta.

Bloco 2 - Matching Típico. Nas 10 tentativas de teste com tarefas de matching típico o participante deveria pegar o sinalizador à esquerda e colocá-lo em baixo de um dos estímulos de comparação à direita (ver Figura $1 \mathrm{~A} \mathrm{e}$ Tabela 2). A resposta de seleção era considerada correta se o palito fosse colocado abaixo do estímulo de comparação idêntico ao estímulo modelo.

Bloco 3 - Matching Adaptado e Matching Típico misturados. O terceiro bloco tinha 20 tentativas de teste com as mesmas tarefas utilizadas no primeiro e no segundo blocos, 10 tentativas para cada tipo de arranjo, porém os dois tipos de tentativas eram misturados ao longo da seqüência. Esse bloco não foi precedido por tentativas de treino (ver Tabela 2) e a seqüência na apresentação dos dois tipos de tarefas não era fixa nem previsível.

Por tratar-se de uma avaliação, não havia consequiências programadas para acerto e erro e nenhum procedimento de ajuda ou correção foi utilizado durante os testes, nos três blocos. Em alguns momentos da sessão eram apresentados elogios e incentivos verbais para manter o participante realizando as atividades até o término da avaliação, porém estas intervenções não eram contingentes a acertos ou erros.

Todas as sessões foram filmadas e o registro do desempenho dos participantes foi realizado a partir dos vídeos. Dois observadores independentes e ingênuos em relação ao propósito da pesquisa registraram $50 \%$ das sessões e a porcentagem de concordância foi de $100 \%$.

\section{Resultados}

A Figura 2 mostra a porcentagem de acertos dos participantes individuais nas tentativas de matching típico ou padrão (barras cinza) e nas tentativas de matching adaptado (barras pretas), quando esses dois tipos de tarefas foram conduzidos separadamente nos Blocos 1 e 2 (painel superior) e no terceiro bloco, quando os dois tipos de tentativas 
eram misturados ao longo de uma seqüência de 20 tentativas (painel inferior).

No primeiro bloco, com tentativas de matching adaptado (ver barras pretas no painel superior), seis participantes $(1,4,9,10,12$ e 19$)$ apresentaram $100 \%$ de acertos, sete $(6,13,14,15,16,17,18)$ obtiveram $60 \%$ ou mais de acertos (mas não atingiram $100 \%$ ) e sete tiveram desempenho abaixo de $60 \%(2,3,5,7,8,11$ e 20$)$. A menor porcentagem de acertos foi $20 \%$ (participantes 11 e 20), próximo do nível de acaso, que era de 33\%. No segundo bloco, no qual foram realizadas tarefas de matching típico (ver barras cinzas no painel superior), três dos participantes que haviam apresentado $100 \%$ de acertos no Bloco 1 mantiveram o desempenho acurado (1, 4 e 19); os outros três apresentaram muitos erros, acertando entre 30 e $60 \%$ das tentativas $(9,10$ e 12). Essa tendência a aumento na quantidade de erros na mudança do tipo de arranjo dos estímulos também foi observada para a maioria dos outros participantes, que apresentaram acertos abaixo de $60 \%$, inclusive cinco participantes $(9,10,12,13$ e 16) que haviam apresentado desempenho acima de $80 \%$ de acertos no matching adaptado, três dos quais passaram a apresentar porcentagem de acertos abaixo de $40 \%$ (próximo do nível do acaso). Finalmente, quatro participantes apresentaram ganhos da ordem de $20 \%$ a $30 \%$ no matching típico, em relação aos seus acertos no matching adaptado (2, 3, 11 e 20). Dois participantes (5 e 8) não realizaram esse bloco por não terem alcançado o critério nas tentativas iniciais de treino. Portanto, uma minoria dos participantes apresentou desempenho acurado em uma ou em ambas as condições de matching de identidade; os demais apresentaram escores intermediários ou baixos, ao nível do acaso e o desempenho mostrou tendência a piorar quando a tarefa mudou de matching adaptado para matching típico.

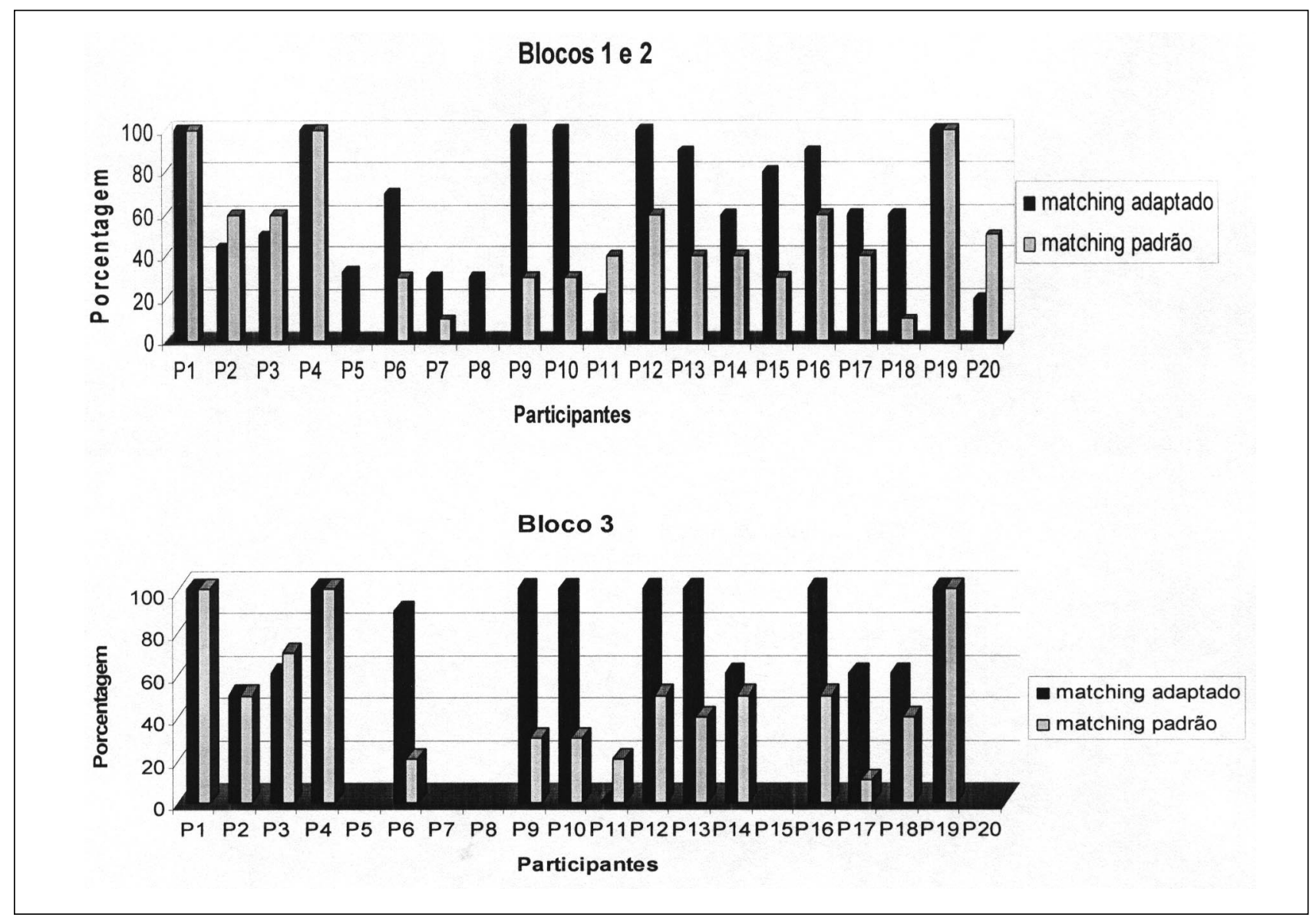

Figura 2. Porcentagem de acertos, de participantes individuais, no matching de identidade com o procedimento adaptado (barras pretas) e com o procedimento típico (barras cinza), nos Blocos 1 e 2, respectivamente (Painel superior) e no Bloco 3, com os dois tipos de tentativas misturadas ao longo da seqüência (Painel inferior)

O terceiro bloco, no qual a apresentação das tentativas de matching típico e de matching adaptado foram misturadas aleatoriamente (ver painel inferior na Figura 2), foi realizado por 15 dos 20 participantes; os outros cinco participantes $(5,7,8,15$ e 20) não fizeram as tarefas desse bloco por não terem conseguido completar o bloco anterior ou por questões comportamentais. Oito dos participantes atingiram 100\% de acertos nas tentativas de matching adaptado: os mesmos seis que também acertaram $100 \%$ no Bloco 1 e dois outros que haviam acertado mais de $80 \%$ naquele bloco (13 e 16); em comparação, no matching típico, apresentaram $100 \%$ de acertos somente os mesmos três 
participantes que já haviam atingido esse nível de acertos no Bloco 2. Seis participantes apresentaram escores na faixa de 40 a $60 \%$, mesmo na condição em que apresentaram os melhores desempenhos. Nove dos quinze participantes apresentaram mais erros nas tentativas de matching típico (quatro que obtiveram $100 \%$ e cinco que obtiveram escores intermediários no matching adaptado) e apenas um participante teve maior porcentagem de acertos no matching típico, em relação ao matching adaptado. De modo geral, apesar da variabilidade entre participantes quanto ao nível de acertos, a tendência nesse bloco foi a mesma observada nos dois primeiros: desempenhos mais precisos e corretos no matching adaptado. Essa tendência ficou mais evidente na Figura 3, que mostra a porcentagem média de acertos nos dois procedimentos, quando apresentados em separado, nos Blocos 1 e 2 (barras à esquerda) ou quando misturados, no Bloco 3 (barras à direita): a média foi de, respectivamente, 44 e 50\% no matching típico e de 67 e $77 \%$ no matching adaptado. Portanto, a média de acertos na situação de matching adaptado foi maior do que na situação de matching típico e a diferença, da ordem de $25 \%$, foi estatisticamente significativa; aplicando-se o Teste de Wilcoxon de Postos Sinalizados, verificou-se a diferença significativa para os Blocos 1 e 2, ao nível de $0,4 \%$ e para o Bloco 3 ao nível de $1,2 \%$. Os dados ainda mostram um pequeno incremento, não significativo, da primeira para a segunda apresentação de cada tipo de procedimento, resultado esse que pode ter sido um artefato gerado pela não inclusão, no Bloco 3, de cinco participantes que tinham apresentado escores muito baixos nos dois primeiros blocos.

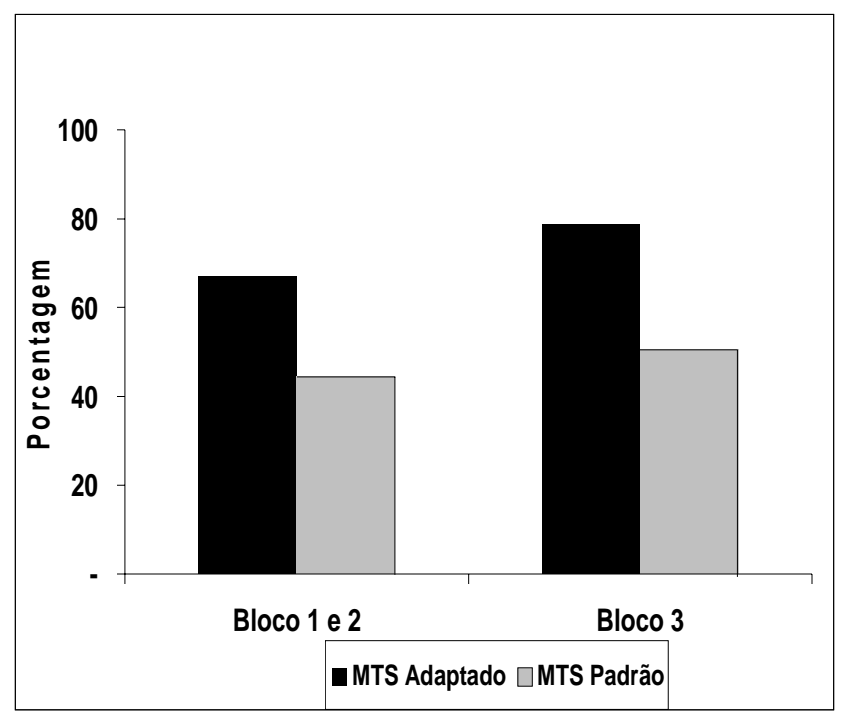

Figura 3. Porcentagem média de acertos nas tarefas de matching com os dois arranjos de estímulos: matching adaptado (barras pretas) e matching típico (barras cinza). Barras à esquerda representam dados nos Blocos 1 e 2 (um para cada tipo de arranjo) e barras à direita representam dados no Bloco 3 (os dois tipos de arranjos misturados ao longo da seqüência de tentativas)

Uma análise mais detalhada das escolhas dos participantes no matching típico, a partir de dados de observação, evidenciou que aproximadamente metade dos participantes $(3,6,7,9,10,11,14,16,18$ e 20$)$ escolheu, sistematicamente, estímulos em uma mesma posição ao longo de tentativas sucessivas, tanto no Bloco 2 quanto no Bloco 3; esse padrão de desempenho resultou em uma probabilidade de acertos ao acaso (33\%) ou próximo do acaso e, portanto, não indica escolha sob controle da igualdade entre os estímulos. A preferência foi maior para a posição do centro (cinco participantes mostraram preferência exclusiva e três mostraram preferência acentuada), seguida pela posição à esquerda (seis participantes). Um exame da distribuição de escolhas de todos os participantes mostrou que a posição à direita foi a menos escolhida (por 55 e $27 \%$ dos participantes, nos Blocos 2 e 3 , respectivamente), e a posição central foi escolhida pelo maior número de participantes $(85 \%$ e $93 \%$ nos Blocos 2 e 3 , respectivamente); a posição à esquerda foi selecionada pelo menos uma vez por $70 \%$ dos participantes no Bloco 2 e por $73 \%$ no Bloco 3 . Nas tentativas de matching adaptado, nos Bloco 1 e 3, não era possível escolher estímulos em uma mesma posição, pois os participantes tinham que distribuir todos os estímulos de comparação sobre os estímulos modelo.

Uma outra análise considerou o desempenho dos participantes nas duas condições em função de algumas de suas características e histórias pessoais. Foram considerados os seguintes aspectos: presença ou não de repertório verbal, tipo de escola freqüentada e classificação na CARS. Os resultados são apresentados na Figura 4. Nessa figura, para cada tipo de variável é apresentada a distribuição percentual de participantes segundo a maior porcentagem de acertos no matching adaptado, maior porcentagem de acertos no matching típico ou porcentagens aproximadamente iguais nos dois tipos de tarefas. Os resultados evidenciam que participantes não verbais, que eram alunos de escolas especiais e obtiveram classificação grave na CARS foram os que apresentaram as maiores discrepâncias nos desempenhos entre os dois tipos de matching, apresentando mais acertos no matching adaptado do que no matching típico. Entre os participantes que apresentaram 100\% de acertos nas duas tarefas, todos apresentavam comportamento verbal desenvolvido e freqüentavam escolas regulares. Os sintomas de autismo desses participantes eram diferentes; o Participante 1 teve pontuação leve/moderado na CARS, o Participante 2 teve pontuação grave, o Participante 4 apresenta Síndrome de Asperger e o Participante 19 apresenta autismo de alto funcionamento.

\section{Discussão}

O objetivo deste estudo foi descrever o desempenho de pessoas diagnosticadas com autismo na realização de uma mesma tarefa, o emparelhamento de identidade com o modelo, organizada de duas maneiras diferentes. A organização se referia ao emprego do matching típico, no qual cada tentativa apresentava um estímulo modelo e três estímulos de comparação, ou do matching adaptado, que apresentava três estímulos modelos e três estímulos de comparação. Esse arranjo é semelhante a tarefas comuns encon- 

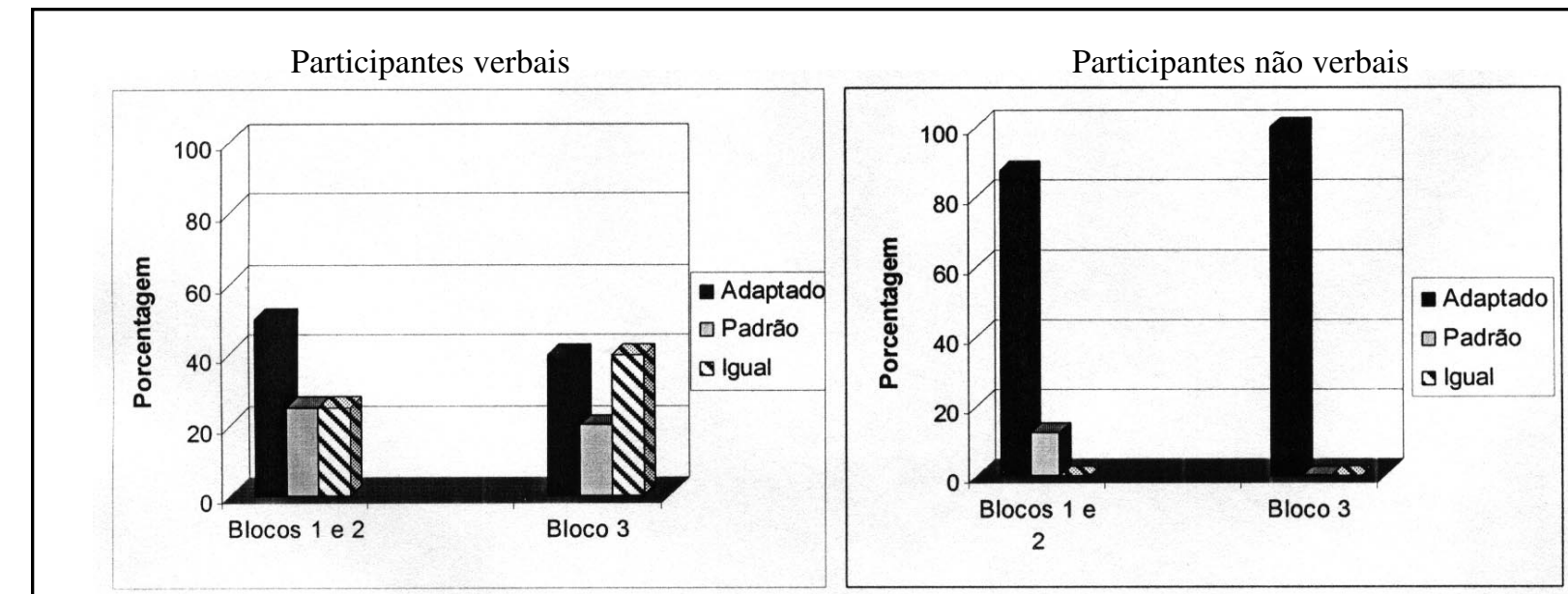

Participantes matriculados em escola regular

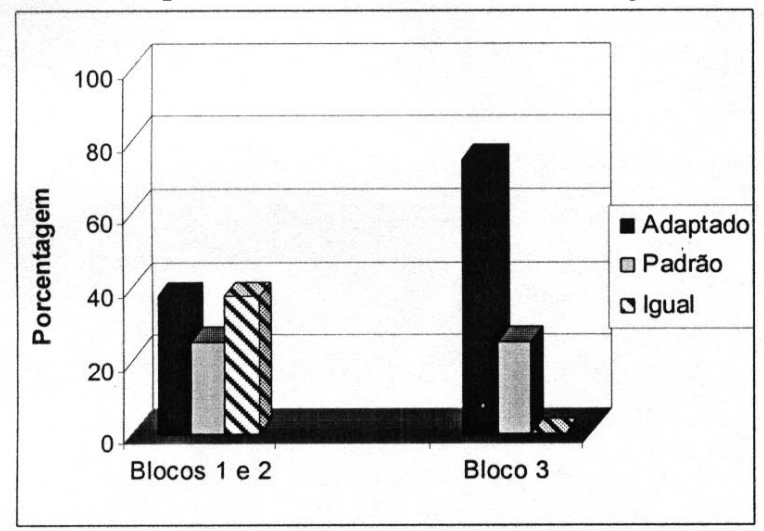

Participantes com CARS leve/moderado

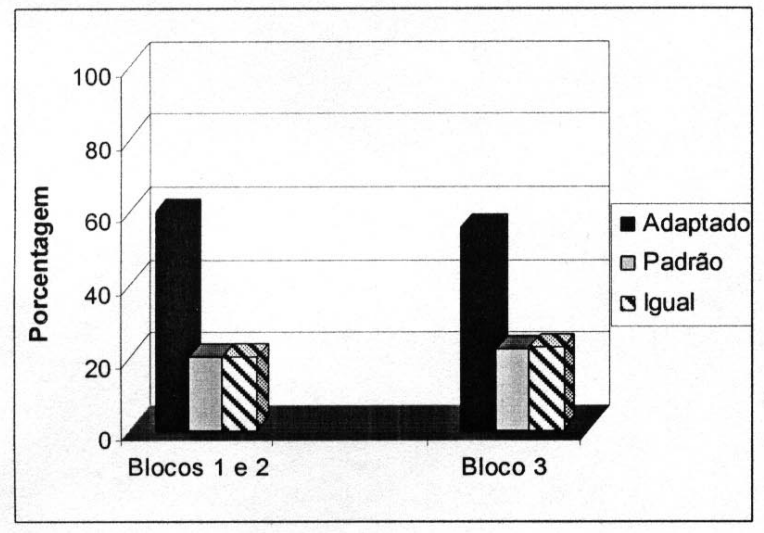

Participantes matriculados em escola especial

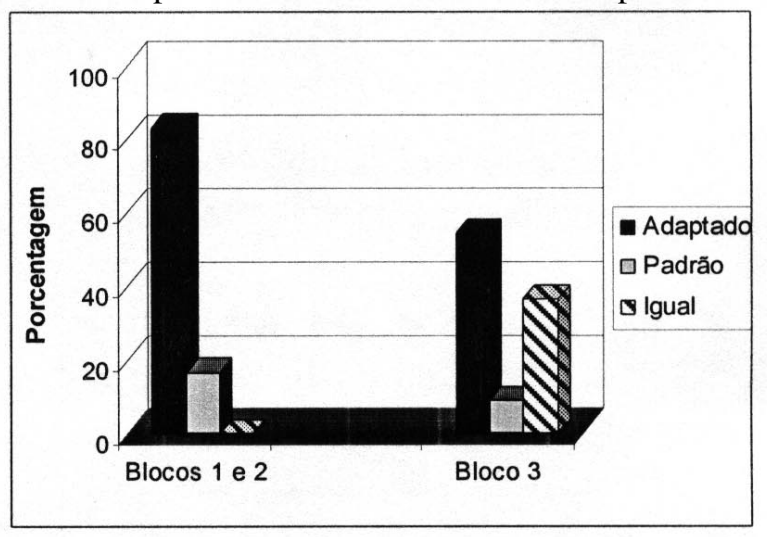

Participantes com CARS grave

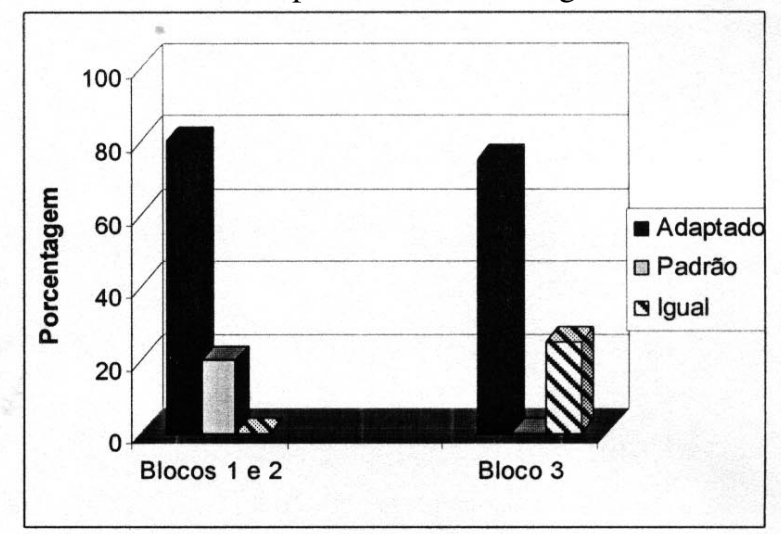

Figura 4. Distribuição porcentual de participantes com mais acertos no matching adaptado, mais acertos no matching típico ou mesmo número de acertos nos dois arranjos, em função do repertório verbal, do tipo de escola e da pontuação na CARS

tradas em livros ou exercícios escolares, quando o aluno tem, por exemplo, que emparelhar elementos ligando os itens correspondentes de dois conjuntos.

Para a finalidade do presente estudo, todos os participantes realizaram ambas as tarefas, primeiro em blocos separados de tentativas para cada tipo de matching e depois em um bloco que misturava esse dois tipos de tenta- tivas. Esse tipo de avaliação de desempenho em duas situações instrucionais diferentes é relevante com pessoas com autismo, pelas peculiaridades destes indivíduos em relação à maneira como se comportam e como aprendem, ressaltadas pela diversificada literatura da área (Frith, 1989; Lovaas, Schreibman, Koegel, \& Rehm, 1971; Peeters, 1998; Rivière, 1995). 
Relatos de dificuldades no ensino de relações condicionais para autistas, seja por emparelhamento de identidade ou por emparelhamento arbitrário, especialmente o auditivo-visual, têm sido comuns (Eikeseth \& Smith, 1992; Kelly et al., 1998; Williams et al., 2005), e a investigação dessas dificuldades, assim como o desenvolvimento de estratégias para remediá-las, é fundamental para que práticas que visam ensinar relações condicionais sejam mais efetivas, requeiram menor número de treinos e resultem em melhor desempenho dos participantes (Dube, 1996). O campo dispõe de uma tecnologia de ensino bastante completa, que parte do repertório do aluno e o desenvolve em pequenos passos ao longo de uma sequiência bem definida a partir de resultados experimentais (Dube, 1996; Serna et al., 1997); e quando, mesmo seguindo pequenos passos o aluno não alcança sucesso em progredir de uma etapa para a outra, a "rota" de treinos dispõe de passos intermediários ainda menores, visando superar essas dificuldades.

Nesse estudo, o objetivo não foi ensinar, mas avaliar o repertório dos indivíduos com autismo em emparelhamento de identidade generalizada e verificar se a maneira pela qual a tarefa é apresentada afeta esse desempenho. Apesar de tratar-se da mesma tarefa, ou seja, tanto no matching típico quanto no matching adaptado o participante tinha que relacionar estímulos de comparação aos estímulos modelos com base em sua similaridade física, a forma de apresentação dos estímulos modelos e comparações, assim como as respostas requeridas dos participantes nos dois tipos de tarefa, eram diferentes, como descrito nas seções precedentes. No matching típico, que tem sido um recurso amplamente utilizado com diversas populações (Gutowski \& Stromer, 2003), para apresentar uma resposta correta o participante precisa olhar para o estímulo modelo, para os estímulos de comparação e indicar apenas uma escolha. No matching adaptado, que é organizado de acordo com critérios do programa TEACCH (1992) e tem sido utilizado em muitas instituições de ensino especializado que atendem pessoas com autismo (Lewins \& de Leon, 1995; Tulimoschi, 2002), o participante deve observar cada estímulo modelo e cada estímulo de comparação e emparelhar cada estímulo de comparação com um dos modelos. Aparentemente, a tarefa de matching adaptado é mais difícil de ser executada por requerer do participante três respostas motoras enquanto o matching típico requer apenas uma; além disso, apresenta dois estímulos a mais para serem examinados e emparelhados, uma vez que a tarefa apresenta três estímulos modelos, e não apenas um.

Embora esta análise pudesse sugerir maior dificuldade dos participantes no matching adaptado, os resultados indicaram que esse arranjo resultou em um desempenho melhor do que no matching padrão ou típico. Muitos participantes que apresentaram uma porcentagem de acertos alta nas tarefas adaptadas, em contraste obtiveram baixa porcentagem de acertos nas tarefas de matching típico. Esses resultados podem refletir os efeitos combinados de uma série de variáveis, algumas das quais relacionadas às características do autismo e às histórias prévias de aprendi- zagem dos participantes do estudo, além das próprias características da organização da tarefa nas duas condições.

Em relação ao autismo, a literatura especializada relata que estas pessoas apresentam dificuldades em aprender por métodos convencionais de ensino, pouca flexibilidade no raciocínio, tendência a manter rotinas, dificuldades frente a situações inesperadas e dificuldades na compreensão de estímulos auditivos, respondendo melhor a estímulos visuais e a situações previsíveis (Lewins \& de Leon, 1995; Peeters, 1998). Descreve-se também respostas idiossincráticas aos estímulos do ambiente e controle restrito de estímulos, demonstrado pela tendência a manter o foco da atenção em detalhes e dificuldades em estabelecer relações entre as partes e o todo (Happé \& Frith, 2006; Lambertuci \& Magalhães, 2005; Lovaas et al., 1971).

Os autores do programa TEACCH, que foi desenvolvido especificamente para educar pessoas com autismo e com alterações na comunicação, afirmam que autistas podem aprender com mais facilidade quando as tarefas são visualmente estruturadas e sugerem três aspectos para essa estruturação: clareza visual, organização visual e instrução visual. Segundo os autores, a clareza visual refere-se aos materiais e modelos utilizados que permitem identificar as características mais importantes da tarefa e reduzir os desvios de atenção. A organização visual refere-se à maneira como a tarefa é organizada, favorecendo que a pessoa compreenda aquilo que deve ser feito. A instrução visual refere-se a aspectos visuais da tarefa que indicam como deve ser feita; por exemplo, onde deve ser iniciada e onde termina (Mesibov et al., 1994).

A maneira pela qual o matching adaptado foi estruturado neste estudo podia favorecer a percepção do início e do término da tarefa pela presença ou ausência de estímulos de comparação na página da esquerda; isto é, enquanto restassem estímulos naquela página, cada um deles deveria ser retirado e colocado sobre o igual na página da direita; quando já não restassem figuras naquela página, a tentativa estava terminada. A disposição dos estímulos também pode favorecer o direcionamento da observação para todos os estímulos, já que requer que cada estímulo de comparação seja colocado sobre um estímulo modelo. Além disso, implica relações visualmente lógicas entre os estímulos (cada estímulo tem seu par). Por outro lado, quando o participante começa a atividade, o primeiro estímulo de comparação que ele coloca sobre um dos estímulos modelos é escolhido entre três estímulos de comparação; já o segundo estímulo de comparação é escolhido entre dois estímulos e no terceiro não há escolha e o participante apenas pega o último estímulo de comparação e o coloca sobre o estímulo modelo restante. Essa característica do procedimento pode favorecer a escolha por exclusão (Dixon, 1977). Contudo a escolha por exclusão não constitui necessariamente um problema. $\mathrm{O}$ responder por exclusão pode ser uma estratégia de ensino eficiente para estabelecer relações entre estímulos (McIlvane \& Stoddard, 1981; McIlvane, Wilkinson, \& de Souza, 2000), especialmente se diferentes estímulos forem a única escolha disponível 
ao longo das tentativas e se essa escolha estiver correta; isto é, se, apesar da escolha ter sido por exclusão, a resposta correta produzir feedback de acerto, esse feedback pode fortalecer a relação entre o estímulo modelo e o estímulo de comparação (Ferrari, de Rose, \& McIlvane, 1993).

As dificuldades em relação ao matching típico podem residir no fato de que a tarefa não necessariamente requer a observação de todos os estímulos e, apesar da presença ou ausência do sinalizador à esquerda poder tornar-se um indicador de início ou término da atividade, a escolha de apenas um dos estímulos de comparação deixa os outros dois "sem par" e, para quem apresenta um pensamento pouco flexível e compreende melhor situações visualmente lógicas, a situação pode indicar que algo está faltando e que a tarefa não terminou. Por não requerer que o participante olhe para todos os estímulos, a tarefa pode levar o participante a observar apenas o sinalizador, apenas os estímulos de comparação ou parte deles, ou ainda somente os pedaços de velcro abaixo das figuras. $\mathrm{O}$ fato de muitos participantes terem mostrado tendência a escolher estímulos em uma mesma posição, especialmente no centro ou na esquerda (mas quase nunca à direita), sugere fortemente que o comportamento ocorreu sob controle da posição: a mais próxima (menor distância entre o palito na página da esquerda e o primeiro estímulo de comparação na página da direita) ou a mais central (uma possível "lógica" diferente da planejada pelo experimentador, que era a relação de identidade entre modelo e comparação). Essas diferentes topografias de controle de estímulos (Dube \& McIlvane, 1996) sugerem, portanto, controle restrito de estímulos, tal como encontrado freqüentemente no ensino de autistas (Litrownik, McInnis, Pritchard, \& Filipelli, 1978; Lovaas, Koegel, \& Schreibman, 1979; Lovaas et al., 1971) e sugerem, principalmente, que o matching típico pode favorecer a ocorrência desse tipo de controle, mais do que o matching adaptado.

Em relação às características dos participantes, os dados indicaram que a maioria dos participantes não verbais, estudantes de escolas especiais e com pontuação grave na CARS, apresentou melhor desempenho no matching adaptado, o que pode sugerir que os autistas mais comprometidos mostram mais dificuldades no matching típico e sendo assim, poderiam se beneficiar mais de estratégias de ensino que utilizem o matching adaptado, pelo menos em uma fase inicial de ensino. É importante considerar, no entanto, que seria fundamental programar o ensino de modo a levar o aluno, gradualmente, a responder sob controle de estímulos apropriado também no matching típico e, desse modo, ampliar seu repertório e sua flexibilidade comportamental; se o indivíduo apresenta rigidez no comportamento e as contingências de ensino incorporam essa característica e não requerem mudanças, a própria história de aprendizagem pode fortalecer ainda mais o padrão de inflexibilidade.

Os autistas verbais, que freqüentam escolas regulares e com pontuação leve/moderado na CARS, apesar de também terem apresentado melhor desempenho no matching adaptado, mostraram-se mais "flexíveis" em relação ao matching típico. Alguns desses participantes até mesmo demonstraram melhor desempenho nesse último tipo de arranjo, enquanto outros apresentaram desempenho similar nos dois tipos de matching. O repertório verbal desenvolvido é claramente produto de história de aprendizagem e a exposição à escola regular pode envolver experiências com tarefas similares à requerida no matching típico. Para esses participantes, o ensino sistemático pelo matching típico, intercalado com o ensino de algumas relações pelo matching adaptado, poderia ser uma estratégia adequada para aprimorar o repertório de identidade generalizada e o repertório conceitual (igual e diferente) e dar início ao ensino de relações arbitrárias. O emparelhamento adaptado, especialmente para matching de identidade, poderia garantir maior densidade de reforçamento e assim funcionar como "tarefa com tentativas fáceis", com a função, sobretudo, de manter o indivíduo engajado na tarefa (alto valor reforçador) e com um repertório de atenção e persistência comportamental que são necessárias para a aprendizagem discriminativa.

A afirmação de Kelly et al. (1998) de que os procedimentos utilizados podem influenciar no desenvolvimento de controle de estímulos encontrou apoio empírico neste estudo de avaliação. O maior número de acertos no matching adaptado sugere que, para a maioria dos participantes deste estudo, com o repertório que apresentavam, esse tipo de organização dos estímulos beneficiou a demonstração de controle de estímulos e poderia favorecer a aprendizagem de novas relações condicionais envolvendo estímulos visuais.

É importante considerar, contudo, que este estudo não pretendeu ensinar e que o reduzido número de tentativas, de fato não ensinou, apenas mediu o desempenho. Portanto, os presentes resultados não podem se comparar aos de estudos que empregaram centenas de tentativas, uma vez que aqueles estudos tinham o objetivo de ensinar relações arbitrárias, que apresentam maior dificuldade que o emparelhamento por identidade.

Outra consideração que deve ser feita é que, na população de 20 participantes, apenas três chegaram a mostrar um repertório de identidade generalizada plenamente desenvolvido, de modo a independer dos arranjos dos estímulos na tarefa; seis apresentaram identidade generalizada apenas sob as condições de matching adaptado, o que sugere a necessidade de condições de ensino adequadas, como comentado antes, para o pleno desenvolvimento desse repertório e o avanço para o estabelecimento de relações arbitrárias entre estímulos, se se pretende promover o desenvolvimento cognitivo, e especialmente o desenvolvimento da função simbólica, fundamental na linguagem, com essa população. Os demais participantes não mostraram emparelhamento generalizado nem sob o matching adaptado; embora seu desempenho tenha sido mais acurado nessa condição, no geral escores ao redor de $60 \%$ ou menos indicam que o repertório não pode ser caracterizado como identidade generalizada e, portanto, esses participantes 
requerem uma programação de ensino sistemática, aplicada de maneira regular e freqüente, para superar os déficits presentes e avançar para repertórios mais avançados.

A natureza dos dados obtidos no presente estudo e o estágio de desenvolvimento do repertório comportamental dos participantes que geraram esses dados sugerem fortemente a necessidade de uma avaliação mais ampla, com participantes com outros repertórios. Por exemplo, com crianças com desenvolvimento típico que apresentem identidade generalizada, os resultados independeriam do tipo de arranjo dos estímulos, como ocorreu para os autistas que apresentaram $100 \%$ de acertos neste estudo? Por outro lado, dadas as dificuldades para a criação de condições adequadas para o ensino de discriminações simples e condicionais com bebês (Bijou, 1957; Gil \& Oliveira, 2003; Gil, Oliveira, Sousa, \& Faleiros, 2006; Oliveira, 2003, 2007; Weisberg \&Rovee-Collier, 1998), poderia o matching adaptado constituir uma etapa inicial e eficaz de ensino? Essas são algumas das questões levantadas pelo presente estudo, cuja investigação empírica poderá contribuir para uma melhor compreensão sobre as condições para o ensino de discriminações como fundamento para a construção de repertórios comportamentais mais complexos não só a indivíduos com autismo, mas também a outras populações que requerem condições especiais de ensino.

\section{Referências}

Annet, J., \& Leslie, J. (1995). Stimulus equivalence class involving olfactory stimuli. The Psychological Record, 45, 439450.

Belanich, J., \& Fields, L. (1999). Tactual equivalence class formation and tactual-to-visual cross modal transfer. The Psychological Record, 49, 75-91.

Bijou, S. W. (1957). Methodology for an experimental analysis of child behavior. Psychological Reports, 3, 243-250.

Catania, A. C. (1999). Aprendizagem: Comportamento, linguagem e cognição. Porto Alegre, RS: Art Med.

DeGrandpre, R. J., Bickel, W. K., \& Higgins, S. T. (1992). Emergent equivalence relations between interoceptive (drug) and exteroceptive (visual) stimuli. Journal of the Experimental Analysis of Behavior, 58, 9-18.

de Rose, J. C. (2004). Emparelhamento com modelo e suas aplicações. In C. N. de Abreu \& H. J. Guilhardi (Eds.), Terapia comportamental e cognitivo-comportamental: Práticas clínicas (pp. 215-225). São Paulo, SP: Roca.

Dixon, L. S. (1977). The nature of control by spoken words over visual stimulus selection. Journal of the Experimental Analysis Behavior, 27, 433-442.

Dube, W. V. (1996). Teaching discrimination skills to persons with mental retardation. In C. Goyos, M. A. Almeida, \& D. G. de Souza (Eds.), Temas em Educação Especial: Programa de Pós-Graduação em Educação Especial: UFSCAR (pp. 73-96). São Carlos, SP: Editora da Universidade Federal de São Carlos.

Dube, W. V., Green, G., \& Serna, R. (1993). Auditory successive conditional discrimination and auditory stimulus equivalence class. Journal of the Experimental Analysis of Behavior, 59, 103-114.
Dube, W. V., \& McIlvane, W. J. (1996). Implications of a stimulus control topography analysis for emergent behavior and stimulus classes. In T. R. Zentall, \& P. M Smeets (Eds.), Stimulus class formation in humans and animals (pp. 197-218). Amsterdam, Netherlands: Elsevier.

Eikeseth, S., \& Smith, T. (1992). The development of functional and equivalence classes in high-functioning autistic children: The role of naming. Journal of the Experimental Analysis of Behavior, 58, 123-133.

Ferrari, C., de Rose, J. C., \& McIlvane, W. J. (1993). Exclusion vs. selection training of auditory-visual conditional relations. Journal of Experimental Child Psychology, 56, 49-63.

Frith, U. (1989). Autism: Explain the enigma. Oxford, UK: Blackwell.

Ghezzi, P., Williams, W., \& Carr, J. (1999). Autism: Behavior analytic perspectives. Reno, NV: Context Press.

Gil, M. S. C. A., \& Oliveira, T. P. (2003). Um procedimento de treino de discriminação condicional com bebês. In M. Z. Brandão, F. C. S. Conte, F. S. Brandão, Y. K. Ingberman, C. B. Moura, V. M. Silva, \& S. M. Oliane (Eds.), Sobre comportamento e cognição: Clínica, pesquisa e aplicação: Vol. 12 (pp. 469-477). Santo André, SP: ESETec.

Gil, M. S. C. A., Oliveira, T. P., Sousa, N. M., \& Faleiros, D. A. M. (2006). Variáveis no ensino de discriminação para bebês. Psicologia: Teoria e Pesquisa, 22, 146-152.

Gutowski, S. J., \& Stromer, R. (2003). Delayed matching to twopicture samples by individuals with and without disabilities: An analysis of the role of naming. Journal of Applied Behavior Analysis, 36(4), 487-503.

Happé, F., \& Frith, U. (2006). The weak coherence account: Detail-focused cognitive style in autism spectrum disorders. Journal of Autism and Developmental Disorders, 36, 5-25.

Hayes, L., Tilley, K., \& Hayes, S. (1988). Extending equivalence class membership to gustatory stimuli. The Psychological Record, 38, 473-482.

Keller, F. S. (1968). "Good-bye teacher . . ." Journal of Applied Behavior Analysis, 1, 79-89.

Kelly, S., Green, G., \& Sidman M. (1998). Visual identity matching and auditory-visual matching: A procedural note. Journal of Applied Behavior Analysis, 31(2), 237-243.

Lambertuci, M., \& Magalhães, L. (2005). Terapia ocupacional nos transtornos invasivos do desenvolvimento. In W. Camargos Jr. (Ed.), Transtornos invasivos do desenvolvimento: $3^{\circ}$ milênio (pp. 227-235). Brasília, DF: CORDE.

Lewins, S. M., \& de Leon,V. C. (1995). Programa TEACCH. In J. S. Schwartzman \& F. B. Assumpção (Eds.), Autismo infantil (pp. 233-263). São Paulo, SP: Memnon.

Litrownik, A. J., McInnis, E. T., Wetzel-Pritchard, A. M., \& Filipelli, D. L. (1978). Restricted stimulus control and inferred attentional deficits in autistic and retarded children. Journal of Abnormal. Psychology, 87, 554-562.

Lovaas, O. I., Koegel, R. L., \& Schreibman, L. (1979). Stimulus overselectivity in autism: A review of research. Psychological Bulletin, 86, 1236-1254.

Lovaas, O. I., Schreibman, L., Koegel, R., \& Rehm, R. (1971). Selective responding by autistic children to multiple sensory input. Journal of Abnormal Psychology, 77, 211-222.

Martin, G., \& Pear, J. (1978). Behavior modification: What it is and how to do it. Englewood Cliffs, NJ: Prentice-Hall.

McIlvane, W. J., \& Stoddard, T. (1981). Acquisition of matchingto-sample performances in severe retardation: Learning by exclusion. Journal of Mental Deficiency Research, 25, 33-48. 
McIlvane, W. J., Wilkinson, K. M., \& de Souza, D. G. (2000). As origens da exclusão. Temas em Psicologia, 8, 195-202.

Mesibov, G. B., Schopler, E., \& Hearsey, A. (1994). Structured teaching. In E. Schopler \& G. B. Mesibov (Eds.), Behavior issues in autism (pp. 193-205). New York: Plenum Press.

Oliveira, T. P. (2003). Aprendizagem de pareamento de identidade por bebês. Dissertação de Mestrado não-publicada, Programa de Pós-Graduação em Educação Especial, Universidade Federal de São Carlos, SP.

Oliveira, T. P. (2007). Contribuições para o controle experimental na aprendizagem de discriminações por bebês. Tese de Doutorado não-publicada, Programa de Pós-Graduação em Educação Especial, Universidade Federal de São Carlos, SP.

Peeters, T. (1998). Autismo: Entendimento teórico e intervenção educacional. Rio de Janeiro, RJ: Cultura Médica.

Rivière, A. (1995). O desenvolvimento e a educação da criança autista. In C. Coll, J. Palácios, \& A. Marchesi (Eds.), Desenvolvimento psicológico e educação: Necessidades educativas especiais e aprendizagem escolar: Vol. 3 (pp. 274-297). Porto Alegre, RS: Artes Médicas.

Saunders, R., \& Green, G. (1999). Discrimination analysis of training-structure: Effects on stimulus equivalence outcomes. Journal of the Experimental Analysis of Behavior, 72, 117-137.

Saunders, K. J., \& Spradlin, J. E. (1989). Conditional discrimination in mentally retarded adults: The effect of training the component simple discriminations. Journal of the Experimental Analysis of Behavior, 52, 1-12.

Saunders, K. J., \& Spradlin, J. E. (1990). Conditional discrimination in mentally-retarded adults - The development of generalized skills. Journal of the Experimental Analysis of Behavior, 54, 239-250.

Schopler, E., Reichler, J. R., \& Renner, C. (1988). CARS-The Childhood Autism Rating Scale. Los Angeles: Westerm Pychological Services.

Serna, R. W., Dube, W. V., \& McIlvane, W. J. (1997). Assessing same/different judgments in individuals with severe intellectual disabilities: A status report. Research in Developmental Disabilities, 18, 343-368.

Sidman, M. (1971). Reading and auditory-visual equivalences. Journal of Speech and Hearing Research, 14, 5-13.

Sidman, M. (1985). Aprendizagem-sem-erros e sua importância para o ensino do deficiente mental. Psicologia, 11(3), 1-15.

Sidman, M. (1994). Equivalence relations and behavior: A research story. Boston: Authors Cooperative.

Sidman, M., \& Tailby, W. (1982). Conditional discrimination $v s$. matching to sample: An expansion of the testing paradigm. Journal of the Experimental Analysis of Behavior, 37, 5-22.

Treatment and Education of Autistic and related Communication handicapped Children. (1992). Independent Tasks: Work Activities for Students with Autism and Other Visual Learners. Chapel Hill, NC: University of North Carolina.

Tulimoschi, M. E. (2002). A psicopedagogia do autismo. Pirassununga, SP: CEDAP.

Weisberg, P., \& Rovee-Collier, C. (1998). Behavioral processes of infants and young children. In A. Lattal \& M. Perone (Eds.), Handbook of research methods in human operant behavior (pp. 325-333). New York: Plenum Press.

Williams, G., Pérez-González, L. A., \& Queiroz, A. (2005). Using a combined blocking procedure to teach color discrimination to a child with autism. Journal of Applied Behavior Analysis, $38,555-558$.

Wing, L. (1988). The continuum of autistic characteristics. In E. Schopler \& G. B. Mesibov (Eds.), Diagnosis and assessment in autism (pp. 91-110). New York: Plenum Press.

Recebido: 27/03/2007 $1^{a}$ revisão: $25 / 09 / 2007$ Aceite final: $26 / 02 / 2008$ 